\title{
High grade endometrial cancer: A prospective cohort study of the outcome of 120 patients after primary surgery
}

\author{
Kodampur $\mathrm{M}^{1, *}$, Papadopoulos $\mathrm{AJ}^{1}$, Montalto $\mathrm{SA}^{1}$, Kopeika $\mathrm{J}^{2}$ and Devaja $\mathrm{O}^{1}$ \\ ${ }^{1}$ West Kent Cancer Centre, Maidstone and Tunbridge Wells NHS Trust \\ ${ }^{2}$ Guy's and St Thomas NHS Trust
}

\begin{abstract}
Objective: the purpose of this study was to assess survival and recurrence site and rate in patients with histological high grade endometrial cancer (grade 3 endometrioid and non endometrioid carcinoma) treated with staging surgery, which included pelvic lymphadenectomy without para aortic lymph node dissection. We have also looked at other histological factors within this group in relationship to survival. Methods: a total of 120 patients diagnosed with high grade endometrial cancer between August 2002 and December 2008 were included in the study. All of them underwent total abdominal (TAH) or laparoscopic assisted vaginal hysterectomy (LAVH) with bilateral salpingo oophorectomy (BSO), with pelvic lymphadenectomy, with or without omentectomy. Results: pelvic lymph node positivity was noted in 37 patients (31\%). The mean follow up was 47 months. Forty-four patients (37\%) developed recurrence with a mean time to relapse of 20 months. The majority of the recurrences (77\%) occurred in both the para aortic area and at distant sites. Among 44 patients with recurrence, 37 (84\%) were lymphovascular invasion (LVSI) positive at primary histology as compared to $41(54 \%)$ patients without recurrence with LVSI $(\mathrm{p}<0.05)$; The proportion of patients with more than $50 \%$ myometrial invasion was much higher among the group with recurrence $(34(77 \%))$ in comparison with those who did not show recurrence $(40$ $(53 \%))(\mathrm{p}<0.05)$. Kaplan-Meier overall survival rate for patients with FIGO stage I was $84 \%$, stage II $70 \%$, stage III $29 \%$ and stage IV $20 \%$. Patients with positive pelvic lymph nodes had a $23 \%$ survival in contrast to $78 \%$ survival without lymph nodal disease (p<0.001). Conclusions: high grade histological subtype of endometrial cancer is a significant risk factor for para aortic lymph nodal involvement and distant relapse. The majority of recurrences of high grade endometrial tumours were distant, which suggests that other additional treatment modalities should be considered in this high risk group.
\end{abstract}

Keywords: endometrial cancer; lymphadenectomy; lymphovascular space invasion; myometrial invasion; recurrence

\section{Introduction}

Uterine cancer is the fourth most common cancer in women, and the ninth most common cause of female cancer death in the United Kingdom [1]. Several factors are known to affect survival including stage and grade. Five-year survival differs dramatically from stage I being $85 \%$, falling to $25 \%$ for women with stage IV disease. Serous, clear cell and other non endometrioid variants (also known as type 2 endometrial cancers) account for $10-20 \%$ of all endometrial cancer cases [2]. Lymph node involvement is an important prognostic factor, and lymphadenectomy is performed for staging purposes, to document the extent of disease spread, and to plan postoperative adjuvant therapy [3].

Several recent publications from Europe have questioned the benefit of this staging procedure $[4,5]$. It is recognised that the risk of lymph node involvement is increased in patients with deep myometrial invasion and high grade disease as demonstrated by Creasman et al. [6] who reported up to $34 \%$ pelvic lymph node metastasis in this group of patients. Mariani et al. [7] reported that external iliac lymph nodes are the most commonly involved nodal group in endometrial cancer. In the UK, staging pelvic lymphadenectomy is more often performed without para aortic nodal dissection. Two large randomised studies

\footnotetext{
*Corresponding author: Kodampur M, West Kent Cancer Centre Maidstone Hospital Maidstone, Kent ME16 9QQ United Kingdom, Tel.: +44 01622224855; Fax: +44 1622224893; E-mail: mkodampur@ doctors.net.uk

Received 12 November 2012 Revised 29 December 2012 Accepted 4 January 2013 Published 11 January 2013

Citation: Kodampur M, Papadopoulos AJ, Montalto SA, Kopeika J, and Devaja $O$ (2013) High grade endometrial cancer: A prospective cohort study of the outcome of 120 patients after primary surgery. J Cancer Res Ther 1: 40-45. doi:10.14312/2052-4994.2013-6

Copyright: (C) 2013 Kodampur M, et al. This is an open-access article distributed under the terms of the Creative Commons Attribution License, which permits unrestricted use, distribution and reproduction in any medium, provided the original author and source are credited.
} 
$[4,5]$ have not shown an improvement in disease free or overall survival with respect to pelvic lymphadenectomy, but other researchers have highlighted shortcomings with these studies [8]. Clearly there is variation in the surgical treatment and staging of endometrial cancer, this varies from no lymphadenectomy to pelvic lymphadenectomy only, through to full pelvic and para aortic lymphadenectomy. It is recognised that for the high grade histological subtypes, lymphadenectomy may play a far more important role in defining adjuvant treatment. It is also true that these patients are often elderly with associated medical morbidity, and extensive surgical staging with pelvic and para aortic lymphadenectomy needs to be justified. Since current common practice in the UK does not include routine para aortic lymphadenectomy [9], we wanted to establish the rate and site of recurrence among patients with high grade endometrial cancer. Even though there are some data on site of recurrence in endometrial cancer $[10,11]$ limited number of them focuses specifically on high grade cancer only $[12,13]$.

The purpose of this study was to assess clinical outcomes including site and rate of recurrence and survival in patients with high grade histological subtypes of endometrial cancer (grade 3 endometrioid carcinoma, serous adenocarcinoma, clear cell adenocarcinoma and endometrial carcinosarcoma malignant mixed Mullerian tumour [MMMT]) following surgery that included pelvic lymphadenectomy without para aortic lymph node dissection (PALND). The effects of other histological characteristics such as LVSI and myometrial invasion were also studied in relationship to above mentioned outcomes.

\section{Methods}

The West Kent Cancer Centre, Maidstone, UK surgically treated 120 consecutive patients with G3 endometrioid adenocarcinoma and non endometrioid type endometrial carcinoma (clear cell adenocarcinoma, serous adenocarcinoma and endometrial carcinosarcoma [MMMT]) from August 2002 to December 2008. All patients had a preoperative radiological stage I, II or IIIC1 without radiologically enlarged para aortic lymph nodes or distant metastases on imaging. Data (imaging and pathology reports, clinical information) was collected prospectively in the Infolex database system (Flex Software Ltd, version 5.40.500). All patients included in this study underwent primary surgery involving total abdominal hysterectomy, bilateral salpingo oophorectomy (TAHBSO) or laparoscopic assisted vaginal hysterectomy, bilateral salpingo oophorectomy (LAVHBSO), with bilateral pelvic lymph node dissection (BPLND) with or without omentectomy. The BPLND entailed removal of tissue overlying the external iliac artery and vein up to the bifurcation of common iliac vessels, tissue in the obturator fossa above the obturator nerve and along the internal iliac vessels. Data on preoperative radiological investigations and histology regarding the lymph node status was collected. For the purpose of this study, the
International Federation of Gynecology and Obstetrics (FIGO) 2009 classification have been reassigned based on the final histology after primary surgery. All patients underwent multidisciplinary meeting discussion of the final histology, and a decision regarding adjuvant therapy was based on associated risk factors such as histological grade, lymph nodal status, LVSI, deep myometrial invasion, cervical/adenexal involvement (FIGO stage). All patients were monitored in the follow up clinic for symptoms or signs of recurrence, and if recurrence suspected further imaging with CT or MRI with histological confirmation were arranged where necessary. The site of recurrence was recorded and the time to recurrence was calculated from the date of surgery to date of recurrence diagnosis. The follow up interval was calculated from the time of primary surgery, until the last clinic visit prior to October 2011. Survival curves were estimated using the KaplanMeier calculation. Statistical significance was determined using the log-rank test and the chi-square test. We regarded $P$ value of less than 0.05 to be as statistically significant. Data analysis was undertaken using PASW Statistics 18 (SPSS Inc, Chicago IL, USA).

\section{Results}

The mean age of all patients was 67 years (range of 42 - 88 years). The mean body mass index was 29 (range of 18 -64). Nulliparity was noted in $19 \%$ of patients. The majority of patients (86\%) presented with post menopausal bleeding. Preoperative imaging with CT and/ or MRI suggested stage I in $79 \%(n=95)$, stage II $11 \%$ $(n=13)$ and stage III $10 \%(n=12)$. The Table 1 shows clinical and pathological characteristics of patients in this study. The mean tumour diameter size was $42.6 \mathrm{~mm}$ (inter quartile range 24.5 - $57.5 \mathrm{~mm}$ ). Lymphovascular space invasion was positively identified in 78 patients $(65 \%)$. The mean number of pelvic lymph nodes removed was 20 (inter quartile range 16 - 45). Pelvic lymph node positivity was established in 37 patients (31\%). Pelvic lymph node metastases were found in $42 \%$ of cases with greater than $50 \%$ myometrial invasion and in $46 \%$ of cases with LVSI. The adnexae were involved in 17 patients (14\%), 82\% of these patients had pelvic nodal metastases. The most common postoperative complication was lymphoedema of the legs (19 patients (15.8\%), In this group of patients 11 out of 19 patients had both pelvic lymphadenectomy and postoperative adjuvant radiotherapy. One patient $(0.8 \%)$ had bowel injury intra operatively. One patient $(0.8 \%)$ had pulmonary embolism. Wound infection was noted in seven cases (5.8\%). A single case of postoperative mortality $(0.8 \%)$ was reported due to worsening of medical co-morbidities on day seven post-op; she had undergone TAHBSO and bilateral pelvic lymphadenectomy.

The mean follow up was 47 months. The recurrence was established in 44 (37\%) patients. Table 2 demonstrates the sites of recurrence for all of these patients. The mean time to relapse was 20 months (inter quartile range 7.75 - 29.5 months). The majority of the recurrences $(77 \%)$ occurred in both the para aortic area and at 
Table 1 The relationship of FIGO stage, histological subtypes, adjuvant therapy and recurrence in high grade endometrial cancer patients

\begin{tabular}{|c|c|c|c|c|c|c|c|}
\hline 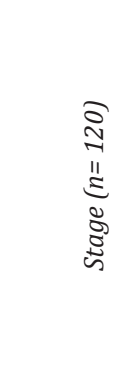 & 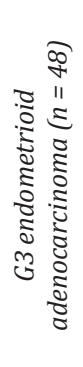 & 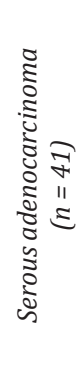 & 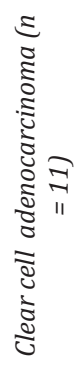 & 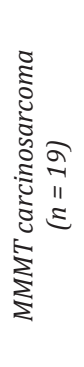 & 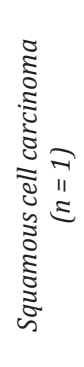 & 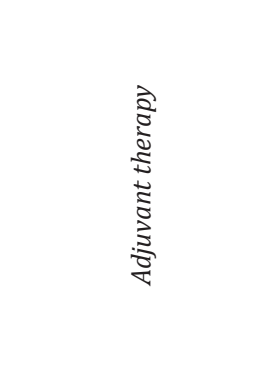 & 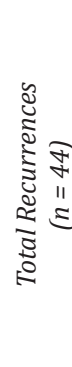 \\
\hline IA (35) & 13 & 15 & 7 & & & 0 & 3 \\
\hline IB (28) & 17 & 8 & 1 & 2 & & $\begin{array}{c}7 \\
\text { (1 EBRT, } 1 \\
\text { EBRT+BRACHY, } \\
5 \text { BRACHY) }\end{array}$ & 8 \\
\hline II (10) & 4 & 1 & 1 & 4 & & $\begin{array}{c}10 \\
\text { (2 EBRT+BRACHY, } \\
7 \text { BRACHY, } \\
1 \text { Combined) }\end{array}$ & 3 \\
\hline IIIA (3) & 3 & & & & & $\begin{array}{c}3 \\
\text { (2 EBRT+BRACHY, } \\
1 \text { Combined) }\end{array}$ & 1 \\
\hline IIIB (7) & 1 & 3 & & 3 & & $\begin{array}{c}6 \\
\text { (1 EBRT, } \\
\text { 2 EBRT+BRACHY, } \\
1 \text { BRACHY, } \\
2 \text { Combined) }\end{array}$ & 4 \\
\hline IIIC1 (32) & 10 & 12 & 2 & 7 & 1 & $\begin{array}{c}26 \\
\text { (4 EBRT, } \\
10 \text { EBRT+BRACHY, } \\
11 \text { Combined, 1 MPA) }\end{array}$ & 21 \\
\hline IV (5) & & 2 & & 3 & & $\begin{array}{c}4 \\
\text { (4 Combined) }\end{array}$ & 4 \\
\hline
\end{tabular}

EBRT $=$ external beam radiotherapy; Brachy = brachytherapy; Combined $=$ radiotherapy + chemotherapy; MPA = medroxy progesterone acetate

distant sites (peritoneum, liver, lung, bowel and bone). Recurrence in the exclusively para aortic area was noted in only one patient. This patient underwent secondary laparotomy and para aortic dissection and survived 70 months as of January 2012. The frequency of recurrence in patients with or without LVSI and myometrial invasion after primary surgery is illustrated in Table 3. Among

Table 2 The various sites of disease in the 44 patients who developed a recurrence in the follow up period

\begin{tabular}{ll}
\hline \multicolumn{1}{c}{ Recurrence site } & $n=44$ \\
\hline Vault only & 8 \\
Para aortic nodes only & 14 \\
Distant* only & 13 \\
Para aortic node + Distant & 2 \\
Para aortic node + Pelvis** & 2 \\
Para aortic node + Pelvic + Distant & 4 \\
Pelvic + Distant & \\
\hline
\end{tabular}

*Distant - recurrences in peritoneum, liver, lung, bowel and/or bones **Pelvis - recurrences in vagina, vault and/or pelvic node
Table 3 The incidence of recurrence in the study group with or without LVSI or $>50 \%$ MI of tumour

\begin{tabular}{|c|c|c|c|c|c|}
\hline 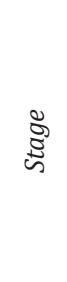 & 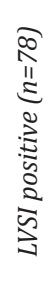 & 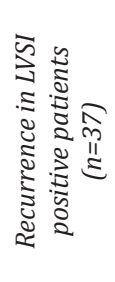 & 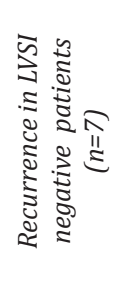 & 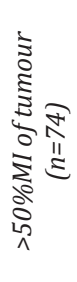 & 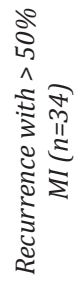 \\
\hline IA & 9 & 2 & 1 & - & - \\
\hline IB & 18 & 6 & 2 & 28 & 8 \\
\hline II & 7 & 1 & 2 & 8 & 2 \\
\hline IIIA & 2 & 1 & 0 & 2 & 1 \\
\hline IIIB & 5 & 2 & 2 & 2 & 5 \\
\hline IIIC1 & 32 & 21 & 0 & 26 & 16 \\
\hline IVB & 5 & 4 & 0 & 5 & 4 \\
\hline
\end{tabular}

LVSI - lymphovascular space involvement MI - myometrial invasion 
the patients with recurrence $(n=44), 84 \%(n=37)$ were LVSI positive in compared with only $54 \%(\mathrm{n}=41)$ of patients without recurrence $(\mathrm{p}<0.05)$. Greater than $50 \%$ myometrial invasion was prevalent in $77 \%(\mathrm{n}=34)$ and $50 \%(n=40)$ in patients with and without recurrence respectively $(\mathrm{p}<0.05)$.

In total 73 women survived and 47 died during this study. The Kaplan-Meier overall survival rate by stage is shown in Figure 1, with FIGO stage I being $84 \%$, stage II $70 \%$, stage III $29 \%$ and stage IV $20 \%$. The Kaplan-Meier curve in Figure 2 represents survival of patients with respect to depth of myometrial invasion by the tumour with greater than $50 \%$ myometrial invasion having a lower survival rate over the study period (48.6\%) than patients with less than $50 \%$ or no myometrial invasion $(80 \%)(\mathrm{p}<0.05)$. Lymphovascular space involvement also significantly $(\mathrm{p}<0.05)$ influenced survival with an overall survival of $51.3 \%$ versus $78.6 \%$ without the presence of LVSI, which is demonstrated in Figure 3. Pelvic lymph node involvement seems to result in the biggest gap in survival (Figure 4). Patients with positive pelvic lymph nodes had a $23 \%$ survival in contrast to a $78 \%$ survival without lymph nodal disease $(\mathrm{p}<0.001)$.

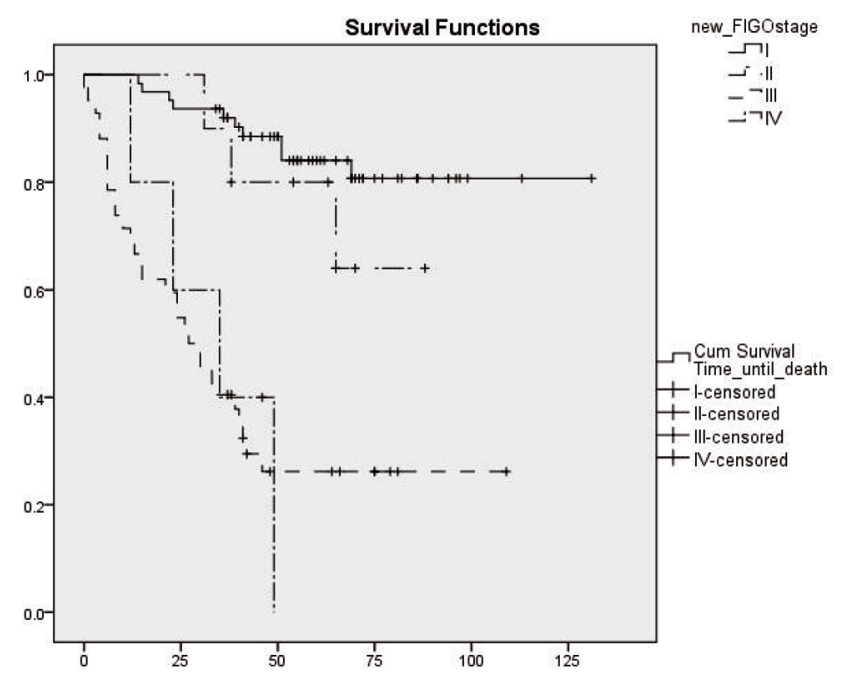

FIGO= international federation of gynecology and obstetrics Time until death- Time from date of surgery to death in months

Figure 1 The Kaplan-Meier overall survival with FIGO stage I-IVB $(\mathrm{P}<0.05)$

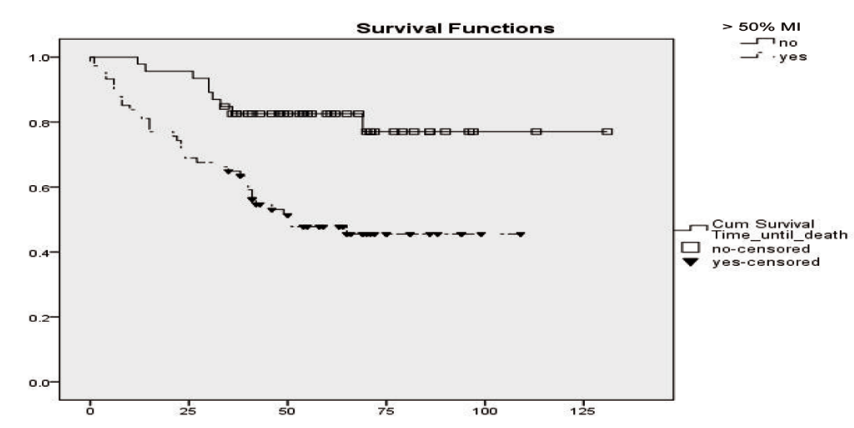

$\mathrm{MI}=$ myometrial invasion

Time until death- Time from date of surgery to death in months

Figure 2 The Kaplan-Meier survival curve of patients with depth of myometrial Invasion $(\mathrm{P}<0.05 \%)$

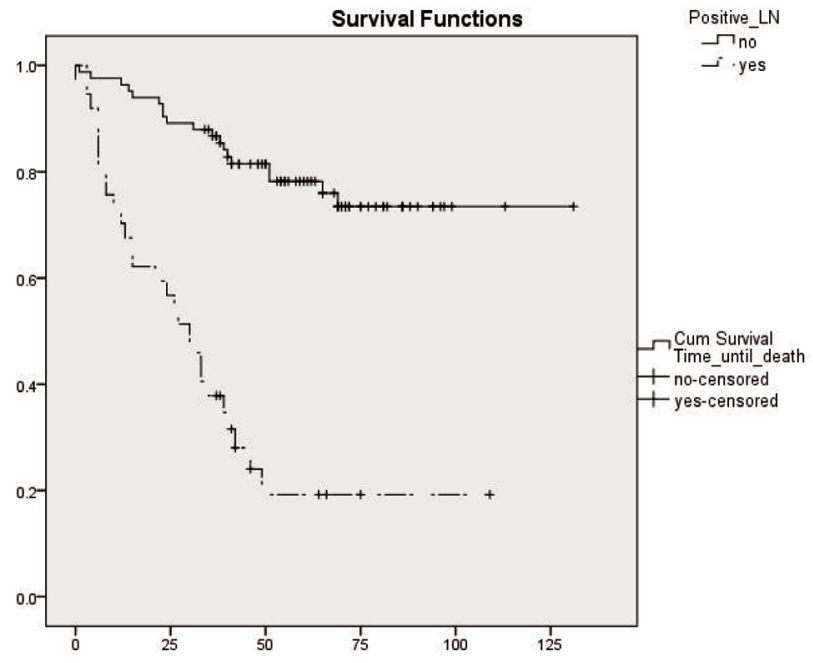

LN= lymph node Time until death- Time from date of surgery to death in months

Figure 3 The Kaplan-Meier survival curve of patients with pelvic lymph node involvement $(\mathrm{P}<0.001)$

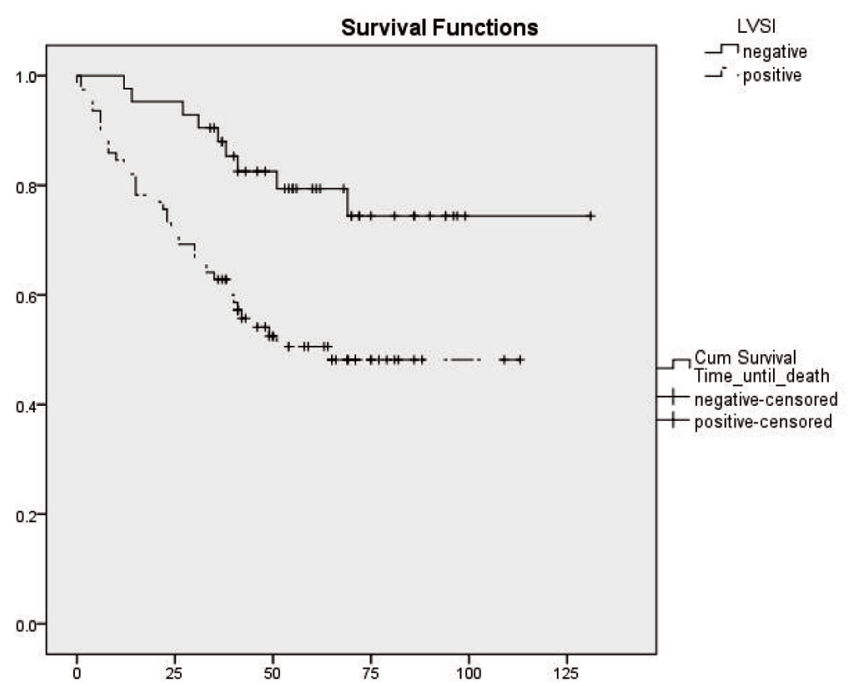

LVSI= lymphovascular space invasion

Time until death- Time from date of surgery to death in months

Figure 4 The Kaplan-Meier survival curve of patients with LVSI involvement $(\mathrm{P}<0.05)$

\section{Discussion}

This study analysed the histological risk factors and clinical outcomes of women with high grade endometrioid (grade 3) and non endometrioid endometrial carcinoma without radiological distant metastases and/or involved para aortic nodes, treated with surgery including pelvic lymphadenectomy, but excluding para aortic nodal dissection. Previously Creasman et al [6] reported lymph node metastasis in more than $40 \%$ of patients with grade 3 tumours with deep myometrial invasion. Our data supports this finding with a $42 \%$ rate of pelvic lymph node metastasis in this group of patients. When the group of patients with pelvic lymph node metastasis was scrutinised it was established that as many as $42 \%$ of patients had LVSI and $46 \%$ of them had myometrial invasion of grater than $50 \%$. However, when we analysed 
the groups of patients with less than 50\% myometrial invasion and LVSI negative, we demonstrated pelvic lymph node metastasis still occurred in $13 \%$ and $5 \%$ of cases respectively.

In the group of patients with recurrence, the para aortic nodes were involved in $41 \%$ ( $\mathrm{n}=18$ of 44 recurrences). Mariani et al [14] reported that $47 \%$ of woman with positive pelvic lymph nodes either had positive para aortic lymph nodes or subsequently experienced a para aortic recurrence. Whilst in our study $67 \%$ of patients with involvedpelvicnodessubsequentlydevelopedarecurrence in the para aortic area. This, on one side, would appear to support the use of para aortic lymph node dissection (PALND) in the treatment of high grade endometrial cancer. However, in our cohort only one patient had an isolated para aortic nodal recurrence, whilst the rest of the para aortic recurrence group had other distant sites involved as well. The overall survival of these patients with para aortic nodal recurrence (these also included distant and pelvic metastases) was 39\%. Lowrey et al [15] reported that G3 endometrioid endometrial cancer, has a significantly better disease specific survival at 5 years when treated with a full staging lymphadenectomy (pelvic and para aortic lymphadenectomy) compared to no lymphadenectomy ( $65.9 \%$ vs. $60.9 \%, \mathrm{p}=0.002)$. In our study, we had 48 patients with grade 3 endometrioid cancer, with slightly higher survival $(75 \%)$ than in the previously mentioned study, despite us not performing a para aortic lymphadenectomy, although we had a smaller number of patients in this group. Our data might suggest that para aortic lymphadenectomy may not necessarily give any survival advantage.

We believe that on balance pelvic lymphadenectomy can provide information which helps in predicting prognosis and planning adjuvant treatment inspite of the current lack of data supporting its therapeutic value. The presence of positive pelvic nodes is known to be an indicator of poor prognosis and the need for adjuvant radiotherapy to the pelvis for better local control. In view of the high percentage of recurrences, which are mainly distant in our study, adjuvant treatment with chemotherapy may be of benefit and further studies are required to assess this. Currently the decision for adjuvant radiotherapy is based on pelvic nodal status, size of the tumour, greater than $50 \%$ myometrial invasion of tumour and LVSI. Johnson et al [16] in their Cochrane review concluded that postoperative platinum based chemotherapy is associated with a benefit in progression-free survival and overall survival irrespective of radiotherapy treatment. Chemotherapy with or without radiotherapy may therefore reduce the chances of developing a distant recurrence. Currently a phase III trial of postoperative chemotherapy or no further treatment for patients with node-negative stage I-II intermediate or high risk endometrial cancer (ENGOTEN2-DGCG / EORTC-55102) is being proposed, to address some of these issues. In addition PORTEC3 a randomised phase III trial comparing concurrent chemoradiation and adjuvant chemotherapy with pelvic radiation alone in high risk and advanced stage endometrial carcinoma is underway and results will be keenly awaited. Our study benefits with results of a single centre standardised surgical protocol and adds to the limited information in this exclusive group of high risk endometrial cancer patients. However we also recognise the limitations of our study. Even though we have focused on high grade cancer only, we still had different histological subtypes that might potentially affect the progression of the disease. The number of patients included in this study is relatively low, but nevertheless it gives a big series coming from individual center with focus on high grade cancer only. Subsequent treatment was also quite heterogeneous and included different modalities such as chemotherapy and radiotherapy chosen on individual basis, therefore the overall survival and recurrence could have been affected by those. In spite of several limitations we believe that the current study has valuable message to relay.

\section{Conclusion}

Our results confirm that high grade histological subtype of endometrial cancer is a significant risk factor for lymph nodal involvement and distant relapse. The majority of recurrences of high grade endometrial tumours were distant, which suggests that systemic adjuvant treatment with chemotherapy is the most logical approach in this high risk group. Further studies are required to assess the role for para aortic lymphadenectomy in the management of high grade histological subtypes of endometrial cancer, as well as addressing the best systemic adjuvant therapy in this setting.

\section{Conflict of interest}

The authors wish to express that they have no financial or other relationships that might lead to a conflict of interest.

\section{References}

[1] Office for National Statistics, Cancer Statistics registrations: Registrations of cancer diagnosed in 2008, England. Series MB1 no.39. 2010, National Statistics: London.

[2] Office for National Statistics Mortality Statistics: Deaths registered in 2010, England and Wales 2011, National Statistics: London.

[3] Morrow CP, Bundy BN, Kurman RJ, Creasman WT, Heller P, et al. (1991) Relationship between surgical-pathological risk factors and outcome in clinical stage I and II carcinoma of the endometrium: a Gynecologic Oncology Group study. Gynecol Oncol 40: 55-65.

[4] ASTEC study group, Kitchener H, Swart AM, Qian Q, Amos C, et al. (2009) Efficacy of systematic pelvic lymphadenectomy in endometrial cancer (MRC ASTEC trial): a randomised study. Lancet 373: 125-36.

[5] Benedetti Panici P, Basile S, Maneschi F, Alberto Lissoni A, Signorelli M, et al. (2008) Systematic pelvic lymphadenectomy vs. no lymphadenectomy in early-stage endometrial carcinoma: randomized clinical trial. J Natl Cancer Ins 100: 1707-1716.

[6] Creasman WT, Morrow CP, Bundy BN, Homesley HD, Graham JE, et al. (1987) Surgical pathologic spread patterns of endometrial cancer. A Gynecologic Oncology Group Study Cancer 60:2035-2041.

[7] Mariani A, Webb MJ, Keeney GL, Podratz KC (2001) Routes of lymphatic spread: a study of 112 consecutive patients with endometrial cancer. Gynecol Oncol 81:100-4. 
[8] Naumann RW (2012) The role of lymphadenectomy in endometrial cancer: was the ASTEC trial doomed by design and are we destined to repeat that mistake? Gynecol Oncol 126: 5-11.

[9] Maggino T, Romagnolo C, Landoni F, Sartori E, Zola P, et al. (1998) An analysis of approaches to the management of endometrial cancer in North America: a CTF study. Gynecol Oncol 68:274-279.

[10] Klopp AH, Jhingran A, Ramondetta L, Lu K, Gershenson DM, et al. (2009) Node-positive adenocarcinoma of the endometrium: outcome and patterns of recurrence with and without external beam irradiation. Gynecol Oncol 115:6-11.

[11] Scarfone G, Secomandi R, Parazzini F, Viganò R, Mangili G, et al. (2013) Clear cell and papillary serous endometrial carcinomas: survival in a series of 128 cases. Arch Gynecol Obstet 287:351356.

[12] Kasamatsu T, Shiromizu K, Takahashi M, Matsumoto K, Shirai $\mathrm{T}$ (1999) Analysis of the initial failure site and spread pattern in endometrial carcinoma: a Japanese experience. Int J Gynecol Cancer 9:470-476.

[13] Nout RA, Smit VT, Putter H, Jürgenliemk-Schulz IM, Jobsen JJ, et al. (2010) Vaginal brachytherapy versus pelvic external beam radiotherapy for patients with endometrial cancer of highintermediate risk (PORTEC-2): an open-label, non-inferiority, randomised trial. Lancet 375:816-823.

[14] Mariani A, Keeney GL, Aletti G, Webb MJ, Haddock MG, et al. (2004) Endometrial carcinoma: paraaortic dissemination. Gynecol Oncol 92: 833-838.

[15] Lowery WJ, Gehrig PA, Ko E, Secord AA, Chino J, Havrilesky LJ, et al. (2012) Surgical staging for endometrial cancer in the elderly - is there a role for lymphadenectomy? Gynecol Oncol 126:12-15.

[16] Johnson N, Bryant A, Miles T, Hogberg T, Cornes P (2011) Adjuvant chemotherapy for endometrial cancer after hysterectomy. Cochrane Database Syst Rev. doi:10.1002/14651858.CD003175.pub2. 